\title{
miR-21 and miR-101 regulate PLAP-1 expression in periodontal ligament cells
}

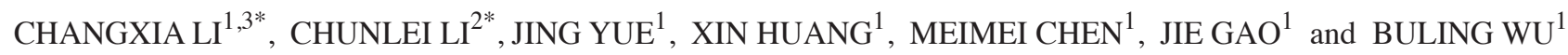 \\ ${ }^{1}$ Department of Stomatology, Nanfang Hospital, Southern Medical University, College of Stomatology, \\ Southern Medical University; ${ }^{2}$ Guangdong Provincial People's Hospital; ${ }^{3}$ Department of Stomatology, \\ The First Affiliated Out-Patient Department Guangzhou Military Area Command, Guangzhou, P.R. China
}

Received December 5, 2011; Accepted February 10, 2012

DOI: $10.3892 / \mathrm{mmr} .2012 .797$

\begin{abstract}
Periodontal ligament-associated protein-1 (PLAP-1/ asporin) is a special marker in periodontal ligament tissue. It is an important regulator of osteogenic differentiation of periodontal ligament cells (PDLCs). This marker is also a prerequisite for periodontal ligament development and mineralization in maintaining homeostasis of the periodontium. However, the molecular mechanisms of the regulation of PLAP-1 expression at the post-transcriptional level remain unknown. By contrast, microRNAs (miRNAs) provide an additional level of regulation beyond that of transcription factors via regulation of the post-transcriptional control of gene expression. This study was designed to analyze miRNA differential expression patterns of PDLCs at various osteoblastic differentiation stages and to determine the contribution of miRNAs in the regulation of PLAP-1 expression during osteoblast differentiation. Bioinformatic analysis was performed to predict miRNAs that potentially regulate the gene expression of PLAP-1. Dual luciferase reporter assay and qRT-PCR were performed to confirm the effects of these miRNAs on PLAP-1 gene expression. Our results indicated that mir-101 and mir-21 target PLAP-1 to regulate its expression during osteogenic differentiation of PDLCs.
\end{abstract}

\section{Introduction}

Periodontal ligament (PDL), the most important tissue influencing the lifespan of the human tooth, is composed of heterogeneous cell populations including osteoblasts, which have osteogenic differentiation potential (1-3). Osteogenesis of periodontal ligament cells (PDLCs) is part of an important

Correspondence to: Dr BuLing Wu, Department of Stomatology, Nanfang Hospital, Southern Medical University, College of Stomatology, Southern Medical University, No. 1838 North Guangzhou Avenue, Guangzhou 510515, P.R. China

E-mail: bulingwu@yahoo.cn

*Contributed equally

Key words: microRNA, periodontal ligament associated protein-1, periodontal ligament cells, gene regulation, osteogenic differentiation process of cytodifferentiation and mineralization to form mineralized tissue cells (4). Osteoblast differentiation results in the expression of tissue-specific gene products that form and regulate the structure of the mineralized PDL extracellular matrix (5).

Periodontal ligament associated protein-1 (PLAP-1/ asporin), a specific marker of the PDL, is an important negative regulator of osteogenic differentiation in maintaining the homeostasis of periodontium (6-8). PLAP-1 was first identified as a novel member of the small leucine-rich repeat proteoglycan (SLRP) family and confirmed to be expressed specifically and predominantly in the PDL (9-10). PLAP-1 was found to be closely associated with the process of cytodifferentiation of PDL cells (11). Therefore, the relative expression level of PLAP-1 may be regarded as a specific marker for the osteogenic differentiation of PDLCs.

Moreover, PLAP-1 was not only expressed specifically in the PDL of an erupted tooth but also in the dental follicle, which is the progenitor tissue of the PDL during tooth development (8). This result suggests that PLAP-1 may be involved in PDL development and cytodifferentiation (11).

Furthermore, it is important to understand the processes that regulate the mineralization of PDLCs. Previous studies showed that the osteogenesis of PDLCs, a complex process in which multiple signaling pathways are involved, is regulated by numerous growth and transcription factors (12-14). However, the mechanism of PLAP-1 expression regulation is not clear, particularly at the post-transcriptional level.

MicroRNAs (miRNAs) provide an additional level of gene regulation beyond that of transcription factors. miRNAs are a class of small non-coding RNA molecules that regulate gene expression post-transcriptionally in eukaryotic cells (15-18). miRNAs are approximately 22 nucleotide single-stranded RNAs that silence gene expression by binding to target mRNAs. Mature miRNAs target the 3' untranslated regions (3'UTR) of genes by complementary base-pair binding (19-20). We hypothesized that certain miRNAs may be able to regulate PLAP-1 gene expression and lead to differentiation of PDLCs.

Therefore, the aim of this study was to determine which miRNAs are responsible for regulating PLAP-1 expression during osteoblast differentiation. We analyzed miRNA differential expression patterns of PDLCs at various osteoblastic differentiation stages. Bioinformatic analysis was performed 
Table I. Oligonucleotides used in this study.

\begin{tabular}{ll}
\hline Primer name & \multicolumn{1}{c}{ Sequence-specific forward primer } \\
\hline has-mir-21 RT & 5'-CTCAACTGGTGTCGTGGAGTCGGCAATTCAGTTGAGTCAACATC-3' \\
has-mir-101 RT & 5'-CTCAACTGGTGTCGTGGAGTCGGCAATTCAGTTGAGTTCAGTTAT-3' \\
U6 RT & 5'-AACGCTTCACGAATTTGCGT-3' \\
PLAP-1-F & 5'-TCAGTCCCAACCAACATTCC-3' \\
PLAP-1-R & 5'-GTGGGACAGATACAGCCTTC-3' \\
OCN-F & 5'-GTGCAGAGTCCAGCAAAGGT-3' \\
OCN-R & 5'-CAACTCGTCACAGTCCGGATT-3' \\
18s rRNA-F & 5'-CCTGGATACCGCAGCTAGGA-3' \\
18s rRNA-R & 5'-GCGGCGCAATACGAATGCCCC-3' \\
hsa-mir-21-F & 5'-ACACTCCAGCTGGGTAGCTTATCAGACTGATG-3' \\
hsa-mir-101-F & 5'-ACACTCCAGCTGGGTACAGTACTGTGATAA-3' \\
miRNA-R & 5'-CTCAACTGGTGTCGTGGA-3' \\
U6-F & 5'-CTCGCTTCGGCAGCACA-3' \\
U6-R & 5'-AACGCTTCACGAATTTGCGT-3'
\end{tabular}

miRNA-R is the universal reverse primer used in conjunction with a sequence-specific forward primer for hsa-mir21 and hsa-mir101. RT, step-loop primer; F, forward; R, reverse.

to predict miRNAs that potentially regulate gene expression of PLAP-1. Dual luciferase reporter assay and qRT-PCR were performed to confirm the effects of these miRNAs on PLAP-1 gene expression.

\section{Materials and methods}

Cell culture of PDLCs. Primary human PDLCs were isolated from explanted healthy PDL in the middle third of the periodontal membrane root of impacted third molars (21). The cells were cultured in a growth medium containing Dulbecco's modified Eagle's medium (DMEM) (Gibco, Grand Island, NY, USA) with $10 \%$ fetal bovine serum, $1 \%$ L-glutamine, $10,000 \mathrm{IU} / \mathrm{ml}$ penicillin $\mathrm{G}, 100,000 \mathrm{mg} / \mathrm{ml}$ streptomycin sulfate and $25 \mathrm{mg} / \mathrm{ml}$ amphotericin $\mathrm{B}$ at $37^{\circ} \mathrm{C}$ with $5 \% \mathrm{CO}_{2}$ in a 24-well plate. Cells from the second passage were used for all experiments. To activate differentiation and mineralization, PDLCs were cultured in a mineralizing medium containing growth medium, $50 \mathrm{mg} / \mathrm{ml}$ ascorbic acid, $10 \mathrm{mM}$ $\beta$-glycerophosphate and $0.01 \mathrm{mM}$ dexamethasone (Sigma, St. Louis, MO, USA) (1-3). 293T cells were grown in DMEM supplemented with $10 \%$ fetal bovine serum.

Mineralization staining. Mineralization of cultured PDLCs was determined using Alizarin Red (AR) staining. Following 14 days of culture in mineralizing medium, the PDLC layer was washed with phosphate-buffered saline (PBS) twice and fixed in $10 \%$ formaldehyde (Sigma-Aldrich) at room temperature for $20 \mathrm{~min}$. The layer was then washed in duplicate with excess $\mathrm{dH}_{2} \mathrm{O}$ prior to the addition of $1 \mathrm{ml}$ of $40 \mathrm{mM}$ AR ( $\left.\mathrm{pH} 4.1\right)$. The plates were incubated at room temperature for $30 \mathrm{~min}$ under gentle agitation. Following removal of the unincorporated dye, the plates were washed twice with PBS and visualized using inverted microscopy (Nikon).
Reverse transcription-PCR. Total RNA was prepared from the mineralized PDLC culture of days 7 and 14. First-strand cDNA synthesis and PCR processes were performed according to the manufacturer's instructions. The primer sets for PCR in this study are summarized in Table I. The reaction conditions were $94^{\circ} \mathrm{C}$ for $5 \mathrm{~min}$, then 30 cycles of amplification; each cycle consisted of $94^{\circ} \mathrm{C}$ for $45 \mathrm{sec}, 51^{\circ} \mathrm{C}$ for $45 \mathrm{sec}$, and $72^{\circ} \mathrm{C}$ for $1 \mathrm{~min}$. The extension time was prolonged to $10 \mathrm{~min}$ in the last cycle.

Bioinformatic analyses. Bioinformatic scans of the 3'UTR of PLAP-1 were conducted using four web-based miRNA target prediction programs: miRanda, PICTAR 4, PITA and TargetScan (22-30). miRNAs were selected based on their targeted prediction by the four programs, conservation of the binding region and the strength of the predicted interaction.

Dual luciferase reporter assay. The psiCHECK ${ }^{\mathrm{TM}}-2$ luciferase vector (Promega, Madison, WI, USA) was used for the dual luciferase assays. The 3'UTR of PLAP-1 was inserted at the XhoI/NotI sites. Fragments of PLAP-1 3'UTR were obtained by PCR using PLAP-1 cDNA as a template. The PCR products were ligated into the psiCHECK-2 vector to construct the plasmid psiPLAP-1 (Promega).

Luciferase assays were performed using the Dual-luciferase reporter assay system (E1910), according to the manufacturer's instructions (Promega). 293T cells were co-transfected with the plasmid psiPLAP-1 $(0.5 \mu \mathrm{g} /$ well $)$ and miRNA $(20 \mu \mathrm{M})$ using Lipofectamine 2000 (Invitrogen). Forty-eight hours after transfection, the medium was removed and the cells were washed twice using PBS. Passive lysis buffer (PLB) $(100 \mu \mathrm{l})$ was added to each well. Cell lysates were collected and luciferase activity was measured by a GloMax ${ }^{\mathrm{TM}}$ 20/20 luminometer (Promega). The luciferase data were expressed 

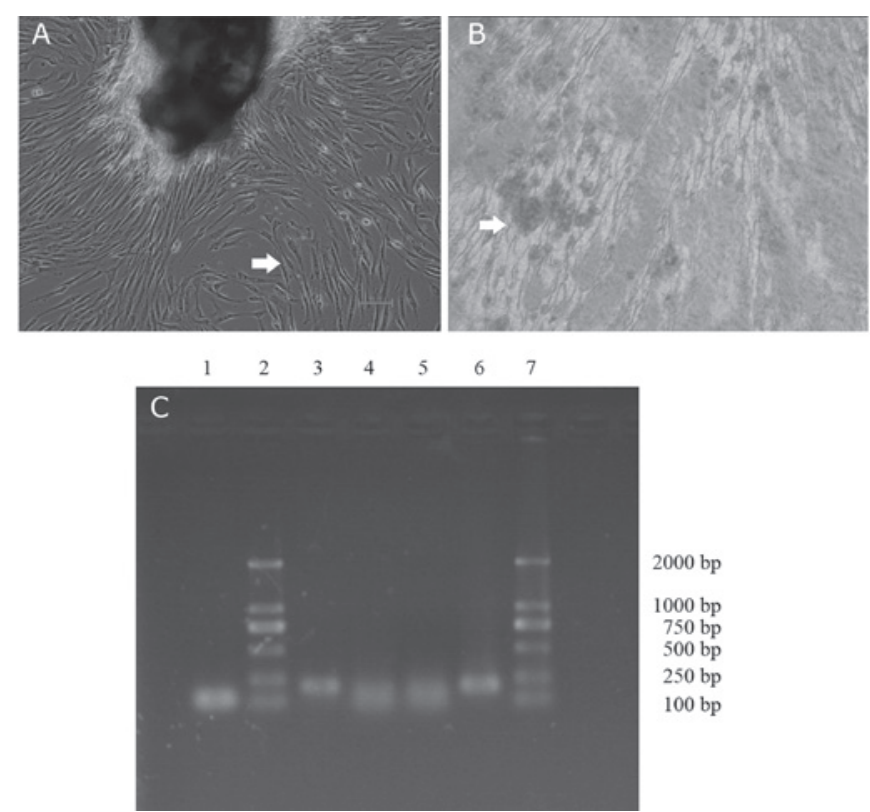

Figure 1. (A) Image of periodontal ligament cells (see arrow) after 10 days of culture. (B) Image of periodontal ligament cells stained by Alizarin Red. After 10 days of culture, mineralized nodules (see arrow) formed.(C) RT-PCR products were separated by gel electrophoresis ( $2 \%$ agarose) and visualized by ethidium bromide staining. PLAP- 1 and OCN were markedly expressed on days 7 and 14 (lane 1, $\beta$-actin; lane 2, 100 bp DNA ladder marker; lane 3, PLAP-1 on day 7, expressed; lane $4, \mathrm{OCN}$ on day 7; lane $5, \mathrm{OCN}$ on day 14 ; lane 6, PLAP-1 on day 14; lane 7, 100 bp DNA ladder marker). PLAP-1, periodontal ligament associated protein-1; OCN, osteocalcin.

as a ratio of Renilla luciferase (RL) to firefly luciferase (FL) to normalize for transfection variability between samples. Luciferase experiments were repeated in triplicate.

$q R T$-PCR. PDLCs were cultured in mineralizing medium to induce osteogenic differentiation. Cells were harvested for isolation of RNA at 7, 10, 14, 21 or 28 days of culture to detect changes in the gene expression of PLAP-1, OCN and two miRNAs. Cells cultured in growth medium served as controls. RNA was isolated from the cells using TRIzol (Invitrogen, Grand Island, NY, USA) according to the manufacturer's instructions. For qPCR, $0.1 \mu \mathrm{g}$ of RNA per reaction was used with the Quantitech SYBR-Green RT-PCR kit and primers specific for PLAP-1 or OCN. To quantify miRNA expression, total RNA was reverse-transcribed for qPCR using the stem-loop method (31-34). Based on the stem-loop feature of the miRNA and cross-species comparison, a number of computational algorithms have been developed to predict miRNAs from the genome. The resulting cDNA was subjected to $\mathrm{qPCR}$ using the universal reverse primer in conjunction with a sequence-specific forward primer of hsa-mir21 and hsa-mir101. Each sample was performed in triplicate, and the results were normalized to 18S rRNA (for PLAP-1) or U6 (for miRNA analysis) (Table I). Results were expressed as fold changes.

Statistical analysis. Data were presented as the mean \pm SD $(n=3)$. Statistical analysis was performed using the Student's t-test. $\mathrm{P}<0.05$ was considered to indicate a statistically significant difference.
Table II. Candidate miRNAs that potentially bind to the PLAP-1 3'UTR.

\begin{tabular}{llll}
\hline miRanda & \multicolumn{1}{c}{ PICTAR 4 } & \multicolumn{1}{c}{ PITA } & \multicolumn{1}{c}{ TargetScan } \\
\hline hsa-mir-144 & hsa-mir-144 & hsa-mir-144 & hsa-mir-144 \\
hsa-mir-101-2 & hsa-mir-101-2 & hsa-mir-101-2 & hsa-mir-101-2 \\
hsa-mir-21 & hsa-mir-21 & hsa-mir-21 & hsa-mir-21 \\
hsa-mir-26a-2 & hsa-mir-26a-2 & hsa-mir-26a-2 & hsa-mir-26a-2 \\
hsa-mir-26b & hsa-mir-26b & hsa-mir-26b & hsa-mir-26b \\
\hline
\end{tabular}

Four miRNA target search algorithms (TargetScan, miRanda, PICTAR 4 and PITA) were utilized to generate a list of candidate miRNAs that potentially bind to the PLAP-1 3'UTR. The miRNAs were predicted using the four programs.

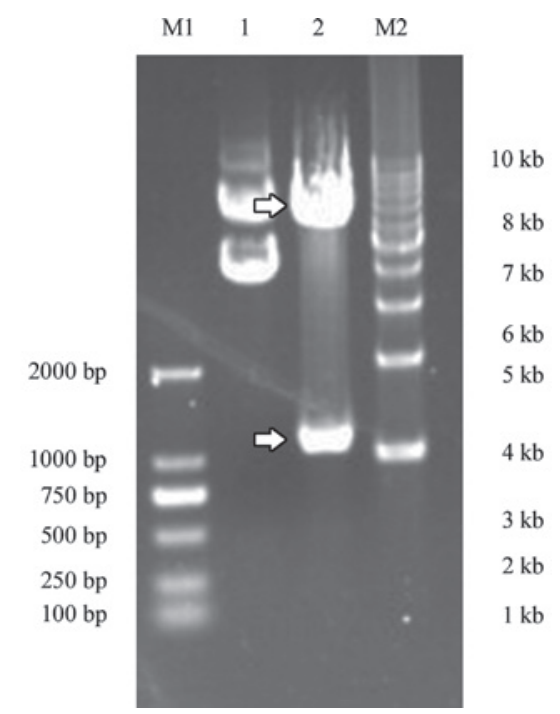

Figure 2. PLAP-1 3'UTR fragment (1086 bp, the arrow below) and the vector fragment $(8.05 \mathrm{~kb}$, the arrow above) following digestion of psiPLAP-1 by XhoI/NotI. M1, DNA marker; lane 1, psiPLAP-1 recombinant plasmid; lane 2, psiCHECK-2 vector inserted with PLAP-1 3'UTR fragment; M2, DNA marker. PLAP-1, periodontal ligament associated protein-1; $3^{\prime}$ UTR, 3 untranslated region.

\section{Results}

Characteristics of PDLCs. Dexamethasone (Dex) and/ or $\beta$-glycerophosphate ( $\beta$-GP) have been shown to induce osteoblast features of PDLCs (1-3). Cultured PDLCs were spindle-shaped in appearance (Fig. 1A). These PDLCs were fibroblast-like cells and were grown to a near-confluent state. After 7 days, mineralized nodules formed and became more condensed. AR staining of mineralized nodules in representative cell cultures is shown in Fig. 1B. Our results showed that two specific markers for the osteogenic differentiation of PDLCs, osteocalcin (OCN) and PLAP-1, were expressed on day 7 and thereafter (Fig. 1C). Previous studies and our data confirmed that PDLCs were a suitable model for our study.

Bioinformatic analyses identified candidate miRNAs that potentially regulate PLAP-1. Based on the stem-loop feature of the miRNA and cross-species comparison, a number of compu- 


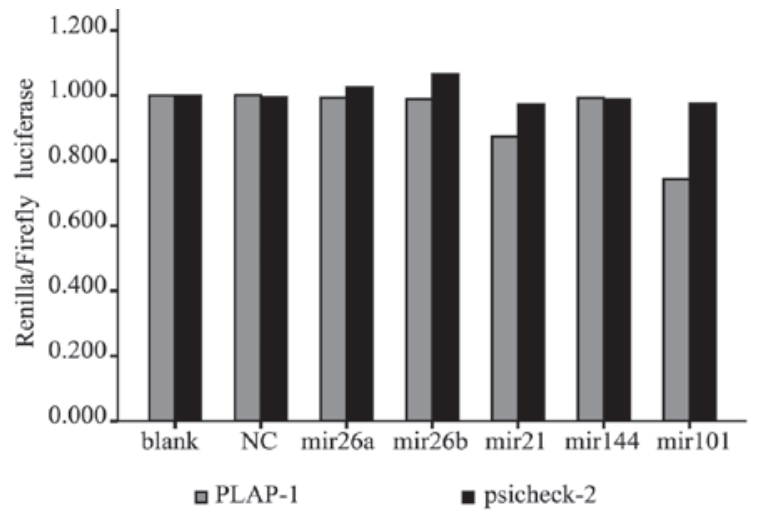

Figure 3. The binding ability of the predicted PLAP-1-targeting miRNAs. Luciferase assays were performed following co-transfection of the PLAP-1 3'UTR luciferase reporter assay and the indicated miRNA precursors. A luciferase signal is shown after normalization to firefly luciferase. Psi is the luciferase vector alone. PsiPLAP-1 is the luciferase vector with a PLAP-1 3'UTR fragment. NC does not code for any known miRNA. PLAP-1, periodontal ligament associated protein-1; 3'UTR, 3' untranslated region.

A

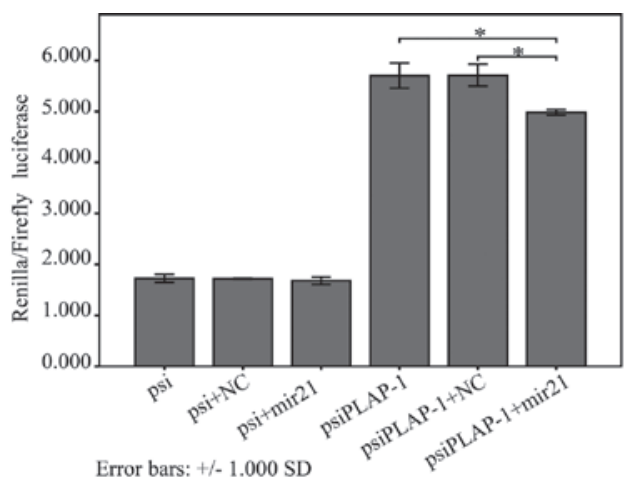

B

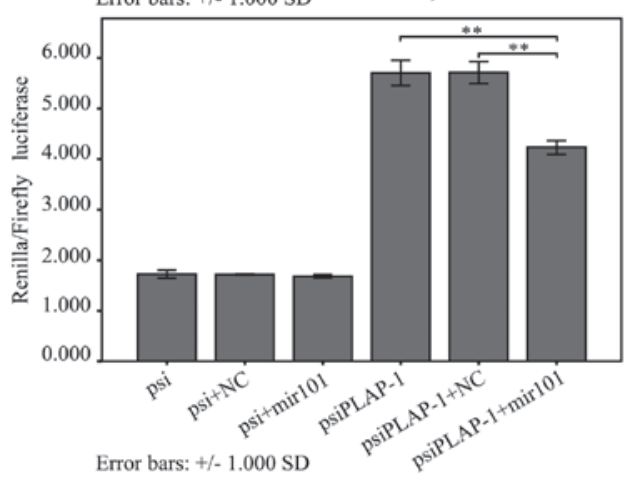

Figure 4. Luciferase reporter assay of (A) mir21 and (B) mir101. Statistical analyses indicate that in the group of cells transfected with the luciferase vector alone, the binding ability of miRNAs was similar to NC (negative control) and blank psi luciferase vector alone. (A) In the group of cells transfected with PLAP-1 3'UTR plasmid, mir21 significantly reduced luciferase activity compared with the blank $\left(\mathrm{P}=0.047, \mathrm{P}=0.041,{ }^{*} \mathrm{P}<0.05\right)$ controls. (B) In the group of cells transfected with the PLAP-1 3'UTR plasmid, mir101 significantly reduced luciferase activity compared with the blank $(\mathrm{P}=0.020$, $\left.{ }^{*} \mathrm{P}<0.05\right)$ and $\mathrm{NC}\left(\mathrm{P}=0.007,{ }^{* *} \mathrm{P}<0.01\right)$ controls. PLAP-1, periodontal ligament associated protein-1; 3'UTR, $3^{\prime}$ untranslated region.

tational algorithms have been developed to predict miRNAs from the genome. Bioinformatic scans are the in silicon standard for assembling a list of candidate miRNAs predicted to target the 3'UTR of a given RNA (22-23,26-27). Four programs (miRanda, PICTAR 4, PITA and TargetScan) were employed
A

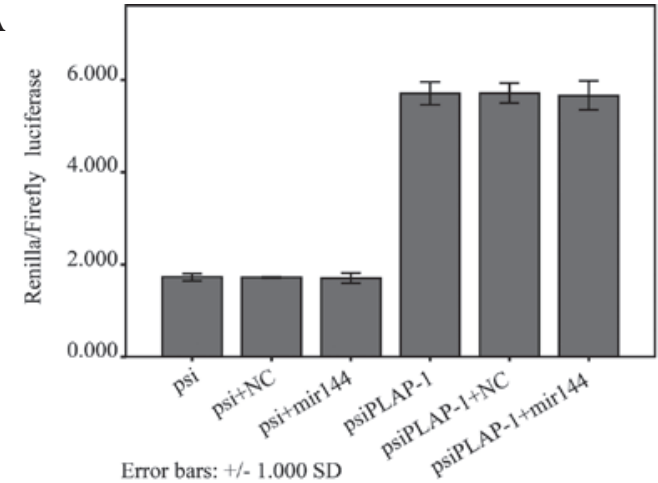

B

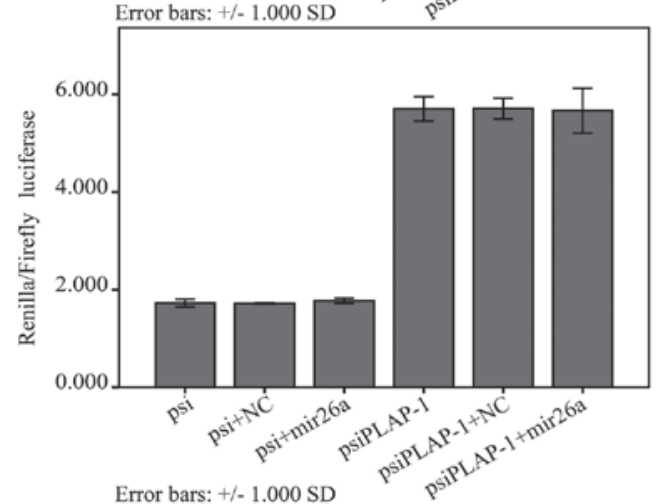

C

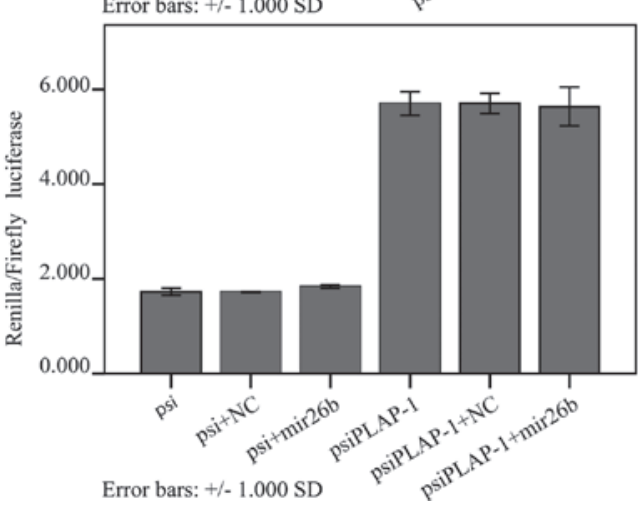

Figure 5. Analysis of luciferase reporter assay for (A) mir144, (B) mir26a and (C) mir26b. Statistical analyses indicate that in the group of cells transfected with the luciferase vector alone, the binding ability of miRNAs was almost identical to that of the NC and blank controls. In the group of cells transfected with the PLAP-1 3'UTR plasmid, three miRNAs did not significantly reduce luciferase activity compared with the blank and NC controls. PLAP-1, periodontal ligament associated protein-1; 3'UTR, 3' untranslated region.

to generate a list of potential miRNAs with a high probability of binding to the 3'UTR of PLAP-1 (Table II). To narrow down this list and increase the probability of identifying miRNAs that are capable of regulating PLAP-1, we tested five miRNAs that were identified by all of the four programs.

The luciferase reporter assay showed that mirl01 and mir21 regulated $P L A P-1$. To test whether candidate miRNAs are capable of interacting with the 3'UTR sequence of PLAP-1, luciferase reporter assays were performed. The 3'UTR of PLAP-1 was inserted into the psiCHECK-2 vector and transiently transfected into $293 \mathrm{~T}$ cells. This construct allowed us to rapidly and quantitatively evaluate miRNA effects on the 3'UTR of PLAP-1. The PLAP-1 3'UTR fragment inserted into the psiCHECK-2 luciferase vector is shown in Fig. 2. The relative luciferase activity in 293T cells transfected with 


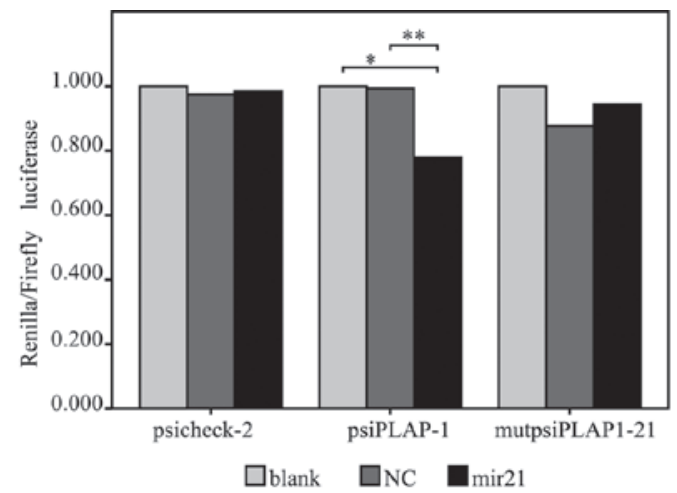

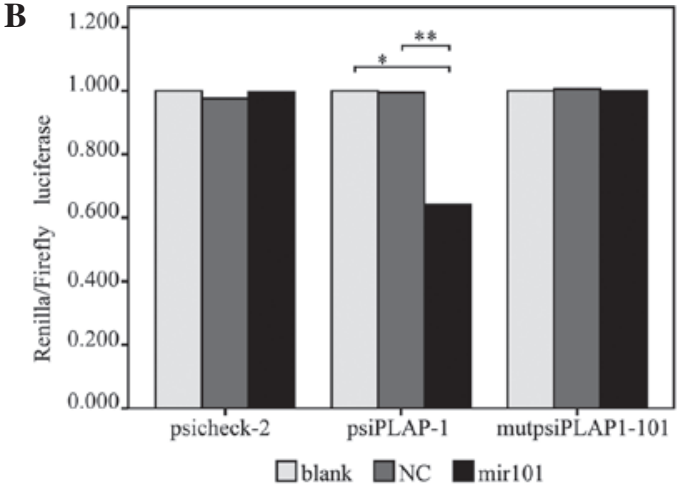

Figure 6. Luciferase reporter assay after mutation of binding sites is shown. The mutation sites were the (A) mir21 binding and (B) mir101 binding sites. The results show that mir21 and mir101 did not reduce luciferase activity following mutation.

A

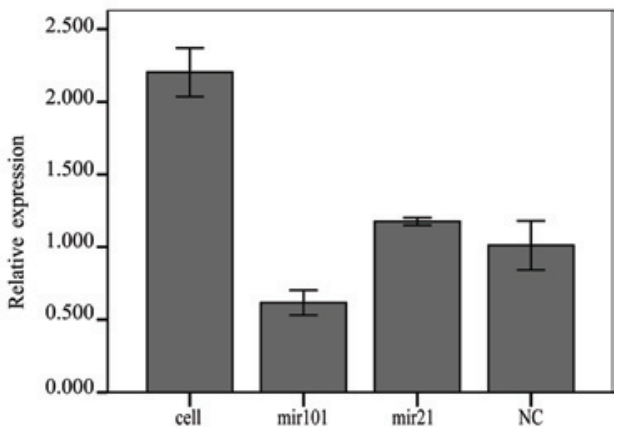

B

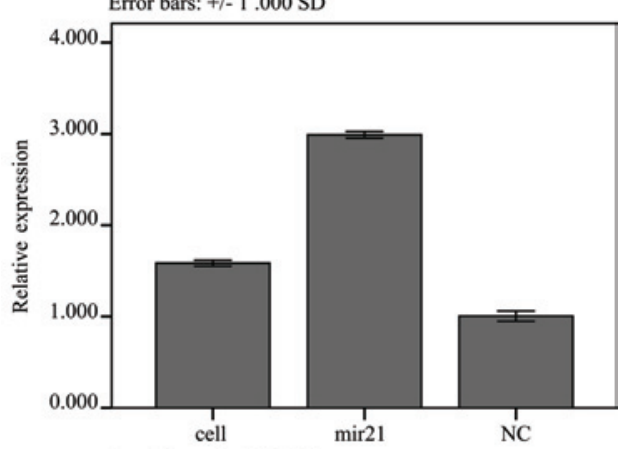

C

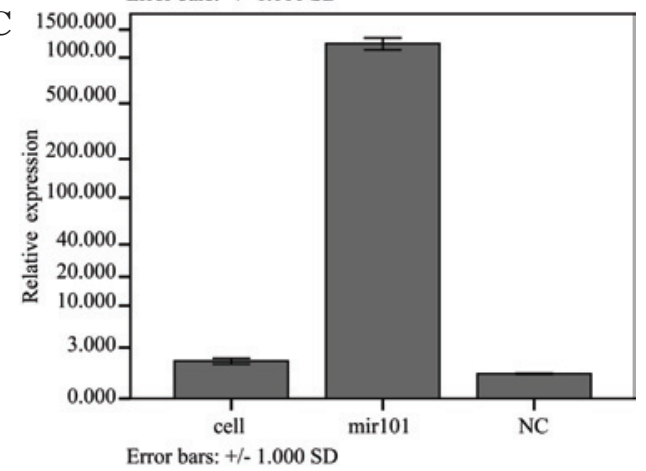

Figure 7. qPCR was used to measure the mRNA level of PLAP-1 following the overexpression of mir21 or mir101. (A) The mRNA level of PLAP-1 was reduced following the overexpression of mir21 or mir101. Overexpression of (B) mir21 and (C) mir101 was confirmed by qPCR. PLAP-1, periodontal ligament associated protein-1.

the luciferase vector alone was arbitrarily set as $100 \%$ for comparison. The binding ability of the five predicted miRNAs with PLAP-1 is shown in Fig. 3. mir101 and mir21 (Fig. 4)
A

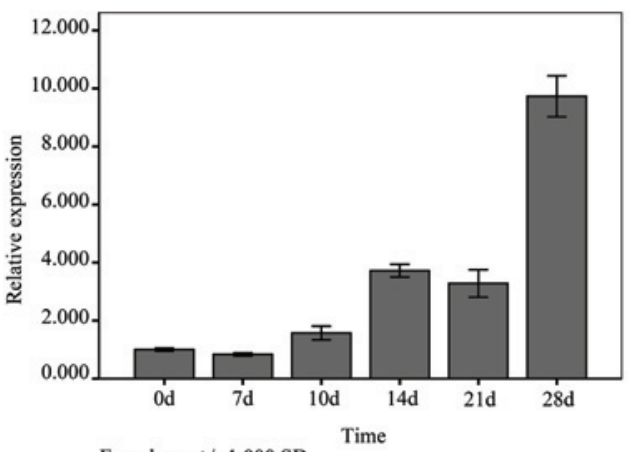

B

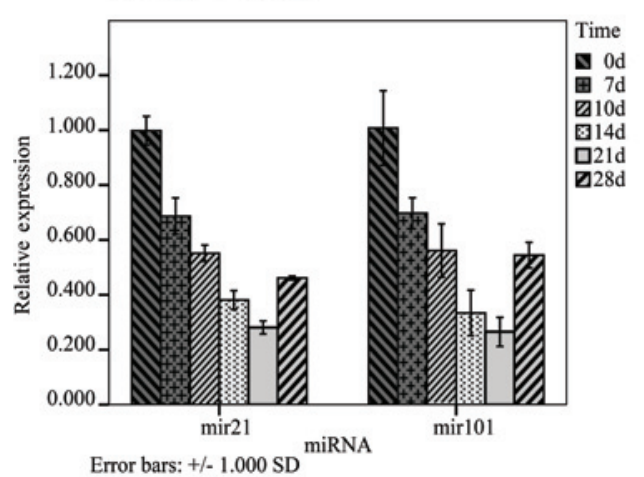

Figure 8. qPCR analysis of (A) PLAP-1 and (B) mir 21 and mir101 at different time points during mineralized culture of PDLCs. (A) Expression of PLAP-1 was low before day 7 and was upregulated thereafter. (B) Expression level of mir21 and mir101 was high initially, but later decreased. PLAP-1, periodontal ligament associated protein-1.

significantly $(\mathrm{P}<0.01, \mathrm{P}<0.05)$ reduced luciferase activity when compared to the negative scrambled miRNA and the luciferase vector alone, whereas mir144, mir26a and mir26b (Fig. 5) did not reduce luciferase activity. Furthermore, we mutated the binding site of PLAP-1 3'UTR to create the constructs mutpsiPLAP-21 and mutpsiPLAP-101. As shown in Fig. 6, mir101 and mir21 were no longer able to reduce luciferase activity. These data suggest that mir101 and mir21 targeted to PLAP-1 to regulate its expression.

Overexpression of mir101 or mir21 reduced the PLAP-1 expression level. To confirm the reporter assay results, mir101 or mir21 were overexpressed and the mRNA level of PLAP-1 was measured. Our qPCR data showed that the overexpression 
of mir101 or mir21 reduced PLAP-1 expression (Fig. 7A). Overexpression of mir21 (Fig. 7B) and mir101 (Fig. 7C) was confirmed by qPCR. These data confirmed that mir101 and mir21 regulated PLAP-1 expression.

Expression level of PLAP-1 was inversely correlated with that of mir21 and mirlo1 during the osteogenic differentiation of PDLCs. PDLCs cultured in mineralizing medium exhibited osteoblastic features, including increased ALPase activities, expression of OCN and calcified nodule formation (35-36). In this study, the expression of PLAP-1 was low prior to day 7 and this expression increased by day 10 . After day 14, PLAP-1 was markedly expressed (Fig. 8A). As a regulator of PLAP-1 gene expression, the expression level of the two miRNAs was inversely correlated with that of PLAP-1 (Fig. 8).

\section{Discussion}

In this study, we described the pattern of PLAP-1/asporin gene expression at various stages of the osteogenic differentiation of PDLCs. Our data have demonstrated that the transcription of PLAP-1 was upregulated during the process of cytodifferentiation of PDL cells into mineralized-tissue-forming cells (Fig. 8A), which is consistent with previous studies (6) Moreover, we established an expression profile of miRNAs targeting the PLAP-1 gene in human PDLCs and identified the differential expression patterns of miRNAs closely associated with the process of osteogenic differentiation of PDLCs for the first time. This study indicates that there is more than one miRNA directly associated with PLAP-1 during the process of osteogenic differentiation. Our data showed that mir101 and mir21 regulated PLAP-1 expression directly. These observations suggest that the expression of PLAP-1 during osteogenesis was controlled not only by differentially expressed transcription factors and growth factors, but also by differentially expressed miRNAs.

This is the first study to explore the effects of miRNAs on PDLC differentiation and PLAP-1 expression. The results have shown that the expression level of PLAP-1 was inversely correlated with that of mir21 and mir101 during the osteogenic differentiation of PDLCs. This finding indicates that mir21 and mir101 may be involved in the osteogenic differentiation of PDLCs. Our aim was not only to identify miRNAs that regulate the PLAP-1 of PDLCs, but also to predict a subset of miRNAs that can be regulated during the differentiation of PDLCs cells and result in abnormal osteogenesis formation. Further investigation in this direction is required.

\section{Acknowledgements}

This study was funded by the College intelligences funds from Guangdong Province (No. C1030270) and the Events of Science and Technology Program of Guang Dong Province (No. 2010B060900053).

\section{References}

1. Shimono M, Ishikawa $\mathrm{T}$, Ishikawa $\mathrm{H}$, et al: Regulatory mechanisms of periodontal regeneration. Microse Res Tech 60: 491-502, 2003
2. Fujii S, Maeda H, Wada N, Kano Y and Akamine A: Establishing and characterizing human periodontal ligament fibroblasts immortalized by SV40T-antigen and hTERT gene transfer. Cell Tissue Res 324: 117-125, 2006.

3. Seo BM, Miura M, Gronthos S, et al: Investigation of multipotent postnatal stem cells from human periodontal ligament. Lancet 364: 149-155, 2004.

4. Ishikawa S, Iwasaki K, Komaki M and Ishikawa I: Role of ascorbic acid in periodontal ligament cell differentiation. $\mathrm{J}$ Periodontol 75: 709-716, 2004.

5. Hess K, Ushmorov A, Fiedler J, Brenner RE and Wirth T: TNFalpha promotes osteogenic differentiation of human mesenchymal stem cells by triggering the NF- $\kappa \mathrm{B}$ signaling pathway. Bone 45: 367-376, 2009

6. Yamada S, Murakami S, Matoba R, et al: Expression profile of active genes in human periodontal ligament and isolation of PLAP-1, a novel SLRP family gene. Gene 275: 279-286, 2001.

7. Yamada S, Ozawa Y, Tomoeda M, Matoba R, Matsubara K and Murakami S: Regulation of PLAP-1 expression in periodontal ligament cells. J Dent Res 85: 447-451, 2006.

8. Yamada S, Tomoeda M, Ozawa Y, et al: PLAP-1/asporin, a novel negative regulator of periodontal ligament mineralization. J Biol Chem 282: 23070-23080, 2007.

9. Lorenzo P, Aspberg A, Onnerfjord P, Bayliss MT, Neame PJ and Heinegard D: Identification and characterization of asporin. a novel member of the leucine-rich repeat protein family closely related to decorin and biglycan. J Biol Chem 276: 12201-12211, 2001.

10. Henry SP, Takanosu M, Boyd TC, et al: Expression pattern and gene characterization of asporin. a newly discovered member of the leucine-rich repeat protein family. J Biol Chem 276: 12212-12221, 2001.

11. Tomoeda M, Yamada S, Shirai H, Ozawa Y, Yanagita M and Murakami S: PLAP-1/asporin inhibits activation of BMP receptor via its leucine-rich repeat motif. Biochem Biophys Res Commun 371: 191-196, 2008.

12. Heikinheimo K: Stage-specific expression of decapentaplegicVg-related genes 2, 4, and 6 (bone morphogenetic proteins 2, 4, and 6) during human tooth morphogenesis. J Dent Res 73: 590-597, 1994.

13. Tucker AS, Al Khamis A and Sharpe PT: Interactions between Bmp-4 and Msx-1 act to restrict gene expression to odontogenic mesenchyme. Dev Dyn 212: 533-539, 1998.

14. Chen Y, Bei M, Woo I, Satokata I and Maas R: Msx1 controls inductive signaling in mammalian tooth morphogenesis. Development 122: 3035-3044, 1996.

15. Ambros V: The functions of animal microRNAs. Nature 431: 350-355, 2004.

16. Bartel DP: MicroRNAs: genomics, biogenesis, mechanism, and function. Cell 116: 281-297, 2004.

17. Lim LP, Glasner ME, Yekta S, Burge CB and Bartel DP: Vertebrate microRNA genes. Science 299: 1540, 2003.

18. Du T and Zamore PD: microPrimer: the biogenesis and function of microRNA. Development 132: 4645-4652, 2005.

19. Place RF, Li LC, Pookot D, Noonan EJ and Dahiya R: MicroRNA-373 induces expression of genes with complementary promoter sequences. Proc Natl Acad Sci USA 105: 1608-1613, 2008.

20. Tay Y, Zhang J, Thomson AM, Lim B and Rigoutsos I: MicroRNAs to Nanog, Oct4 and Sox 2 coding regions modulate embryonic stem cell differentiation. Nature 455: 1124-1128, 2008.

21. About I, Bottero MJ, de Denato P, Camps J, Franquin JC and Mitsiadis TA: Human dentin production in vitro. Exp Cell Res 258: 33-41, 2000.

22. Thomas LF, Saito T and Saetrom P: Inferring causative variants in microRNA target sites. Nucleic Acids Res 39: e109, 2011.

23. Sahoo S and Albrecht AA: Ranking of microRNA target prediction scores by Pareto front analysis. Comput Biol Chem 34: 284-292, 2010.

24. Gamazon ER, Im HK, Duan S, et al: Exprtarget: an integrative approach to predicting human microRNA targets. PLoS One 5: e13534, 2010

25. Xia W, Cao G and Shao N: Progress in miRNA target prediction and identification. Sci China C Life Sci 52: 1123-1130, 2009.

26. Volinia S, Visone R, Galasso M, Rossi E and Croce CM: Identification of microR NA activity by Targets' Reverse EXpression. Bioinformatics 26: 91-97, 2010.

27. John B, Enright AJ, Aravin A, Tuschl T, Sander C and Marks DS: Human MicroRNA targets. PLoS Biol 2: e363, 2004. 
28. John B, Sander C and Marks DS: Prediction of human microRNA targets. Methods Mol Biol 342: 101-113, 2006.

29. Griffiths-Jones S, Saini HK, van Dongen S and Enright AJ: miRBase: tools for microRNA genomics. Nucleic Acids Res 36: D154-D158, 2008.

30. Lewis BP, Burge CB and Bartel DP: Conserved seed pairing, often flanked by adenosines, indicates that thousands of human genes are microRNA targets. Cell 120: 15-20,2005.

31. Fiedler SD, Carletti MZ and Christenson LK: Quantitative RT-PCR methods for mature microRNA expression analysis. Methods Mol Biol 630: 49-64, 2010.

32. Chen C, Tan R, Wong L, Fekete R and Halsey J: Quantitation of microRNAs by real-time RT-qPCR. Methods Mol Biol 687: 113-134, 2011.
33. Chen C, Ridzon DA, Broomer AJ, et al: Real-time quantification of microRNAs by stem-loop RT-PCR. Nucleic Acids Res 33: e179, 2005.

34. Huang X, Xu S, Gao J, et al: miRNA expression profiling identifies DSPP regulators in cultured dental pulp cells. Int J Mol Med 28: 659-667, 2011

35. Kitagawa M, Ueda H, Iizuka S, et al: Immortalization and characterization of human dental pulp cells with odontoblastic differentiation. Arch Oral Biol 52: 727-731, 2007.

36. Nomiyama K, Kitamura C, Tsujisawa T, et al: Effects of lipopolysaccharide on newly established rat dental pulp-derived cell line with odontoblastic properties. J Endod 33: 1187-1191, 2007. 